In the degenerate case when $y=0$ the general result (4), (5), becomes

$$
\int_{-\infty}^{\infty}\left[c^{2 k}+\left(x-x^{-1}\right)^{2 k}\right]^{-1} d x=\pi k^{-1} c^{1-2 k} \csc (\pi / 2 k) \text {. }
$$

Special results for $k=1, c=\frac{1}{2}$ and $k=2, c=1 / \sqrt{2}$ are

$$
\int_{0}^{\infty} \frac{d x}{1+\left(x-x^{-1}\right)^{2}}=\int_{0}^{\infty} \frac{d x}{1+\left(x-x^{-1}\right)^{4}}=\pi \text {, }
$$

which may be verified independently.

Dalhousie University, Halifax, N. S., Canada

Naval Research Establishment, Dartmouth, N. S., Canada

1. A. Erdelti, ET AL., Tables of Integral Transforms, McGraw-Hill, New York, 1954. 1957.

2. F. ObenhemtinaER, Tabellen zur Fourier Transformationen, Springer-Verlag, Berlin,

3. A. VAN der Zigi, Noise, Prentice-Hall, Inc., New York, 1954, p. 316.

\title{
On the Evaluation of Certain Complex Elliptic Integrals
}

\author{
By H. A. Lang and D. F. Stevens
}

1. Introduction. Elliptic integrals of the third kind are occasionally encountered in the form

$$
a \Pi\left(\phi, \alpha^{2}, k\right)+\bar{a} \Pi\left(\phi, \bar{a}^{2}, k\right)
$$

or

$$
a \Pi\left(\phi, \alpha^{2}, k\right)-\bar{a} \Pi\left(\phi, \alpha^{2}, k\right)
$$

where $a, \bar{a}$ and $\alpha^{2}, \bar{\alpha}^{2}$ are complex conjugates and the modulus $k$ is real such that $0<k^{2}<1.0$. It is usually desirable to rewrite these integrals using only real coefficients and parameters. This paper gives an elementary procedure for the evaluation of these expressions, together with the correction of some existing formulas.

2. Modified Development. We follow the development suggested by Hoüel [1], but use the notation of Byrd and Friedman [2] wherever applicable. Since the modulus $k$ is the same in all of the elliptic integrals considered here, it will be omitted throughout.

For convenience, set

$$
\begin{aligned}
& 2 \Pi_{1}=\Pi\left(\phi, \alpha^{2}\right)+\Pi\left(\phi, \bar{\alpha}^{2}\right)=\int_{0}^{\phi} \frac{d \phi}{\left(1-\alpha^{2} \sin ^{2} \phi\right) \Delta}+\int_{0}^{\phi} \frac{d \phi}{\left(1-\bar{\alpha}^{2} \sin ^{2} \phi\right) \Delta} \\
& 2 i \Pi_{2}=\Pi\left(\phi, \alpha^{2}\right)-\Pi\left(\phi, \alpha^{2}\right)=\int_{0}^{\phi} \frac{d \phi}{\left(1-\alpha^{2} \sin ^{2} \phi\right) \Delta}-\int_{0}^{\phi} \frac{d \phi}{\left(1-\bar{\alpha}^{2} \sin ^{2} \phi\right) \Delta}
\end{aligned}
$$

Received August 24, 1959. 
where

$$
\Delta=\sqrt{1-k^{2} \sin ^{2} \phi}
$$

Then, if $a=a_{1}+2 b_{1}$, we have

$$
\begin{aligned}
& a \Pi\left(\phi, \alpha^{2}\right)+\tilde{a} \Pi\left(\phi, \bar{\alpha}^{2}\right)=2 a_{1} \Pi_{1}-2 b_{1} \Pi_{2} \\
& a \Pi\left(\phi, \alpha^{2}\right)-\bar{a} \Pi\left(\phi, \alpha^{2}\right)=2 i a_{1} \Pi_{2}+2 i b_{1} \Pi_{1} .
\end{aligned}
$$

$\Pi_{1}$ and $\Pi_{2}$ will be given by the equations

$$
\begin{aligned}
& s_{1} \Pi_{1}+t_{1} \Pi_{2}=-\frac{1}{m_{1}} F(\phi)-n_{1} \Pi\left(\phi, \alpha_{1}^{2}\right)+\tau_{1} \\
& s_{2} \Pi_{1}+t_{2} \Pi_{2}=-\frac{1}{m_{2}} F(\phi)-n_{2} \Pi\left(\phi, \alpha_{2}^{2}\right)+\tau_{2}
\end{aligned}
$$

where

$$
\tau_{i}=\int_{0}^{p_{i}} \frac{d x}{1+h_{i} x^{2}}, \quad p_{i}=\frac{\sin \phi \cos \phi}{\left(1+m_{i} \sin ^{2} \phi\right) \Delta}
$$

and the other subsidiary quantities are obtained as follows. We first set $\alpha^{2}=-\gamma_{1}-$ $i \gamma_{2}, r^{2}=\gamma_{1}^{2}+\gamma_{2}^{2}, z=\sin ^{2} \phi$ in equations (1) and rationalize:

$$
\begin{aligned}
& \Pi_{1}=\int_{0}^{\phi} \frac{\left(1+\gamma_{1} z\right) d \phi}{\left(1+2 \gamma_{1} z+r^{2} z^{2}\right) \Delta} \\
& \Pi_{2}=\int_{0}^{\phi} \frac{-\gamma_{2} z d \phi}{\left(1+2 \gamma_{1} z+r^{2} z^{2}\right) \Delta}
\end{aligned}
$$

We substitute these forms in a general equation (3) to obtain*

$$
\begin{aligned}
\int_{0}^{\phi} \frac{s\left(1+\gamma_{1} z\right) d \phi}{\left(1+2 \gamma_{1} z+r^{2} z^{2}\right) \Delta}-\int_{0}^{\phi} \frac{t \gamma_{2} z d \phi}{\left(1+2 \gamma_{1} z+r^{2} z^{2}\right) \Delta} & +\frac{1}{m} \int_{0}^{\phi} \frac{d \phi}{\Delta} \\
& +n \int_{0}^{\phi} \frac{d \phi}{\left(1-\alpha^{2} z\right) \Delta}=\int_{0}^{p} \frac{d x}{1+h x^{2}}
\end{aligned}
$$

Differentiating both sides of $(4)$ with respect to $\phi$ we obtain

$$
\frac{s\left(1+\gamma_{1} z\right)-t z \gamma_{2}}{\left(1+2 \gamma_{1} z+r^{2} z^{2}\right) \Delta}+\frac{1}{m \Delta}+\frac{n}{\left(1-\alpha^{2} z\right) \Delta}=\frac{1}{1+h p^{2}} \frac{d p}{\lambda^{2}} \text {. }
$$

The left side of this equation reduces to

$$
\frac{a_{0}+a_{1} z+a_{2} z^{2}+a_{3} z^{3}}{b_{0}+b_{1} z+b_{2} z^{2}+b_{3} z^{3}} \cdot \frac{1}{\Delta}
$$

where

$$
a_{0}=s+\frac{1}{m}+n \quad b_{0}=1
$$

* The two sets of subsidiary quantities arise from the two routs of a quadratic equation in $m$ which results from this general equation. Note that the $\alpha$ in equation (4) is a generic expression for the $\alpha_{1}$ and $\alpha_{3}$ of equations (3), and is therefore distinct from the $\alpha$ of equations (1) and (2). 


$$
\begin{array}{ll}
a_{1}=s \gamma_{1}-t \gamma_{2}-\frac{\alpha^{2}}{m}+\frac{2 \gamma_{1}}{m}-s \alpha^{2}+2 n \gamma_{1} & b_{1}=2 \gamma_{1}-\alpha^{2} \\
a_{2}=-s \alpha^{2} \gamma_{1}-t \alpha^{2} \gamma_{2}+n r^{2}-\frac{2 \gamma_{1} \alpha^{2}}{m}+\frac{r^{2}}{m} & b_{2}=r^{2}-2 \gamma_{1} \alpha^{2} \\
a_{3}=-\frac{\alpha^{2} r^{2}}{m} & b_{3}=-\alpha^{2} r^{2} .
\end{array}
$$

The right side of $(5)$ reduces to

where

$$
\frac{c_{0}+c_{1} z+c_{2} z^{2}+c_{3} z^{2}}{d_{0}+d_{1} z+d_{2} z^{2}+d_{3} z^{3}} \cdot \frac{1}{\Delta}
$$

$$
\begin{array}{ll}
c_{0}=1 & d_{0}=1 \\
c_{1}=-(2+m) & d_{1}=2 m-k^{2}+h \\
c_{2}=k^{2}(2 m+1) & d_{2}=m^{2}-2 m k^{2}-h \\
c_{3}=-k^{2} m & d_{3}=-k^{2} m^{2} .
\end{array}
$$

Setting $a_{i}=c_{i}, b_{i}=d_{i}$ we obtain the following relations:

$$
\begin{aligned}
& \text { (I) } s+\frac{1}{m}+n=1 \\
& \text { (II) } s \gamma_{1}-t \gamma_{2}-\frac{\alpha^{2}}{m}+\frac{2 \gamma_{1}}{m}-s \alpha^{2}+2 n \gamma_{1}=-(2+m) \\
& \text { (III) }-s \alpha^{2} \gamma_{1}+t \alpha^{2} \gamma_{2}+n r^{2}-\frac{2 \gamma_{1} \alpha^{2}}{m}+\frac{r^{2}}{m}=k^{2}(2 m+1) \\
& \text { (IV) } \alpha^{2} r^{2}=m^{2} k^{2} \\
& \text { (V) } 2 \gamma_{1}-\alpha^{2}=2 m-k^{2}+h \\
& \text { (VI) } r^{2}-2 \gamma_{1} \alpha^{2}=m^{2}-2 m k^{2}-h .
\end{aligned}
$$

The elimination of $h$ from (V) and (VI) and the use of (IV) results in a quadratic in $m$ with real roots:

$$
\left(r^{2}+2 k^{2} \gamma_{1}+k^{2}\right) m^{2}+2\left(1-k^{2}\right) r^{2} m-r^{2}\left(r^{2}+2 \gamma_{1}+k^{2}\right)=0 .
$$

Letting $m_{1}, m_{2}$ be these roots, we solve equations (6) for all subsidiary quantities as follows

$$
\begin{aligned}
\alpha_{i}{ }^{2} & =\frac{m_{i}{ }^{2} k^{2}}{r^{2}} \\
s_{i} m_{i} & =m_{i}-m_{i} n_{i}-1 \\
n_{i} m_{i} & =\frac{m_{i}\left[\alpha_{i}{ }^{4}-\left(2+m_{i}\right) \alpha_{i}{ }^{2}+(1+2 m) k^{2}\right]-r^{2}}{r^{2}+2 \gamma_{1} \alpha_{i}{ }^{2}+\alpha_{i}{ }^{4}} \\
t_{i} m_{i} \gamma_{2} & =m_{i}{ }^{2}+\left(\gamma_{1}+2-\alpha_{i}{ }^{2}\right) m_{i}+n_{i} m_{i}\left(\gamma_{1}+\alpha_{i}{ }^{2}\right)+\gamma_{1} \\
h_{i} & =\frac{\left(\alpha_{i}{ }^{2}-k^{2}\right)\left(k^{4}+2 k^{2} \gamma_{1}+r^{2}\right)}{k^{2}\left(1-k^{2}\right)}
\end{aligned}
$$


If we take $m_{1}^{2}<m_{2}^{2}$ then $0<\alpha_{1}^{2}<k^{2}<\alpha_{2}^{2}<1$ and $h_{1}<0<h_{2}$. In the special case where $r^{2}+2 \gamma_{1}+k^{2}=0$, we have $m_{1}=0=\alpha_{1}^{2}$ and $h_{1}=-r^{2}$. Then the first equation of $(3)$ is replaced by

$$
s_{1} \Pi_{1}+t_{1} \Pi_{2}=-\frac{k^{2}}{r^{2}} F(\phi)+\frac{1}{r} \tanh ^{-1} \frac{r \cos \phi \sin \phi}{\Delta}
$$

the second remaining unchanged.

3. Formulas to be Corrected. It will be seen that the definitions (7) are those given by Byrd and Friedman under formulas 417.00 and 438.00 [2, p. 231, 232, 238] with the exceptions of $s_{i}$ and $t_{i}$. Thus the formulas depending upon these quantities are incorrect. They are $416.00,417.00,418.00,419.00,437.00,438.00$, 439.00 and 440.00 .

We also note that these same errors occurred in Hoüel [1, equations 119, p. LIII]. He gives, in his own notation

$$
\begin{aligned}
A & =(1-C) g-1 \\
C g\left(\mu^{2}-2 m \mu \cos \nu+m^{2}\right) & =g\left[m^{2}+(2+g) m+(1+2 m) k^{2}\right]-\mu^{2} .
\end{aligned}
$$

These equations should read respectively

$$
\begin{gathered}
A g=(1-C) g-1 \\
C g\left(\mu^{2}-2 m \mu \cos \nu+m^{2}\right)=g\left[m^{2}+(2+g) m+(1+2 g) k^{2}\right]-u^{2} .
\end{gathered}
$$

To correct Byrd and Friedman formulas 416.00, 417.00, 419.00, 437.00, 438.00, and 440.00 it is necessary only to modify the definitions of $s_{i}$ and $t_{i}$ to those listed in (7), since as listed they are formal consequences of equations ( 3 ) and equation (8). In formulas 418.00 and 439.00 , however, we must not only modify $s_{i}$ and $t_{i}$, but also change the sign of the term involving $\Pi\left(\alpha_{i}{ }^{2}, k\right)$ (in both 418.00 and 439.00), and the sign of the term involving $\tanh ^{-1}\left(r s n u_{1} c d u_{1}\right)$ (in 439.00); these signs should all be negative. (See the end of the next section for a modified, corrected version of 439.00.)

4. Simplication of Results. The form of definitions (7) suggests the following simplifications in the Byrd and Friedman formulas under consideration. Let us multiply equations (3) by $m_{i}$ to obtain

$$
s_{i} \Pi_{1}+t_{i} \Pi_{2}=-F(\phi)-n_{i} \Pi\left(\phi, \alpha_{i}{ }^{2}\right)+\tau_{i} \quad i=1,2
$$

where now

$$
\tau_{i}=\int_{0}^{p_{i}} \frac{m_{i} d x}{1+h_{i} x^{2}}
$$

the $p_{i}$ are defined as before, and definitions (7) are replaced by:

$$
\begin{aligned}
\alpha_{i}^{2} & =\frac{m_{i}{ }^{2} k^{2}}{r^{2}} \\
s_{i} & =m_{i}-n_{i}-1 \\
n_{i}\left(r^{2}+2 \gamma_{1} \alpha_{i}{ }^{2}+\alpha_{i}{ }^{4}\right) & =m_{i}\left[\alpha_{i}^{4}-\left(2+m_{i}\right) \alpha_{i}{ }^{2}+(1+2 m) k^{2}\right]-r^{2}
\end{aligned}
$$




$$
\begin{aligned}
i_{i} \gamma_{2} & =m_{i}^{2}+m_{i}\left(\gamma_{1}+2-\alpha_{i}^{2}\right)+n_{i}\left(\gamma_{1}+\alpha_{i}^{2}\right)+\gamma_{1} \\
h_{i} k^{2}\left(1-k^{2}\right) & =\left(\alpha_{i}^{2}-k^{2}\right)\left(k^{4}+2 k^{2} \gamma_{1}+r^{2}\right) .
\end{aligned}
$$

This permits us to write formulas $416.00,417.00,437.00$ and 438.00 with no explicit. appearances of $m_{1}$ or $m_{2}$. For example, 416.00 becomes:

$$
\begin{aligned}
416.00^{\prime}: a \Pi\left(\alpha^{2}\right)+a \Pi\left(\alpha^{2}\right)= & \frac{2}{s_{1} t_{2}-s_{2} t_{1}}\left\{\left[a_{1}\left(t_{1}-t_{2}\right)+b_{1}\left(s_{1}-s_{2}\right)\right] K\right. \\
& \left.+n_{2}\left(a_{1} t_{1}+b_{1} s_{1}\right) \Pi\left(\alpha_{2}^{2}\right)-n_{1}\left(a_{1} t_{2}+b_{1} s_{2}\right) \Pi\left(\alpha_{1}^{2}\right)\right\}
\end{aligned}
$$

A similar simplification is possible in the special case when $m_{1}=0=\alpha_{1}^{2}$. In this case we multiply the second equation of (3) by $m_{2}$ as above, and multiply equation (8) by $r^{2}$ to obtain

$$
s_{1} \Pi_{1}+t_{1} \Pi_{2}=-k^{2} F(\phi)+r \tanh ^{-1} \frac{r \cos \phi \sin \phi}{\Delta}
$$

where

$$
\begin{aligned}
s_{1} & =r^{2}-k^{2} \\
t_{1} \gamma_{2} & =2 r^{2}+r^{2} \gamma_{1}+k^{2}
\end{aligned}
$$

$\left(n_{2}, s_{2}\right.$ and $t_{2}$ are defined as in $\left(7^{\prime}\right)$.) This permits us to write formulas 418.00, $419.00,439.00$, and 440.00 with no explicit appearances of $m_{2}$ or $r_{2}$ (But note that the two occurrences of $r$ in formulas 439.00 and 440.00 are preserved.) For example, 439.00 (with the sign changes mentioned in Section 4 incorporated) becomes

$$
\begin{aligned}
439.00^{\prime}: a \Pi\left(u_{1}, \alpha^{2}\right)+\bar{a} \Pi\left(u_{1}, \bar{\alpha}^{2}\right) & =\frac{2}{s_{2} t_{1}-t_{2} s_{1}}\left\{\left[a_{1}\left(k^{2} t_{2}-t_{1}\right)+b_{1}\left(k^{2} s_{2}-s_{1}\right)\right] u_{1}\right. \\
& -n_{2}\left(a_{1} t_{1}+b_{1} s_{1}\right) \Pi\left(u_{1}, \alpha_{2}{ }^{2}\right)+\left(a_{1} t_{1}+b_{1} s_{1}\right) \tau^{2} \\
& \left.-r\left(a_{1} t_{2}+b_{1} s_{2}\right) \tanh ^{-1} \frac{r \cos \phi \sin \phi}{\Delta}\right\} .
\end{aligned}
$$

The Rand Corporation

Santa Monica, California

1. G. J. HoüzL, Recueil de Formules et de Tables Vumériques, Gauthier-Villars. Paris, 1901

2. Paul F. BYrd \& Morris D. FriedMan, Handbook of Elliptic Integrals for Engineers and Physicists, Springer-Verlag, Berlin, 1954.

\section{The Numerical Evaluation of the Eighteenth Perfect Number}

\section{By D. Scheffler and R. Ondrejka}

On November 17, 1959 the IBM 709 installation at the National Aviation Facilities Experimental Center in Atlantic City, New Jersey computed the largest known perfect number, corresponding to the eighteenth Mersenne prime [1]. The result was checked by recomputation one week later. Running time for this cal-

Received December 2, 1959. 